\title{
Intersections between polyvictimisation and mental health among adolescents in five urban disadvantaged settings: the role of gender
}

Mphatso Kamndaya ${ }^{1,2}$, Pedro T. Pisa ${ }^{1}$, Matthew F. Chersich ${ }^{1}$, Michele R. Decker ${ }^{3}$, Adesola Olumide ${ }^{4}$, Rajib Acharya ${ }^{5}$, Yan Cheng ${ }^{6,7}$, Heena Brahmbhatt ${ }^{1,3}$ and Sinead Delany-Moretlwe ${ }^{1 *}$

\begin{abstract}
Background: Polyvictimisation (PV) - exposure to violence across multiple contexts - causes considerable morbidity and mortality among adolescents. Despite high levels of violence in urban disadvantaged settings, gender differences in associations between PV and mental health have not been well established.

Methods: We analysed data from a survey with 2393 adolescents aged 15-19 years, recruited using respondentdriven sampling from urban disadvantaged settings in Baltimore (USA), Delhi (India), Ibadan (Nigeria), Johannesburg (South Africa) and Shanghai (China). PV was defined as exposure to two or more types of violence in the past 12 months with family, peers, in the community, or from intimate partners and non-partner sexual violence. Weighted logistic regression models are presented by gender to evaluate whether PV is associated with posttraumatic stress, depression, suicidal thoughts and perceived health status.

Results: PV was extremely common overall, but ranged widely, from $74.5 \%$ of boys and $82.0 \%$ of girls in Johannesburg, to 25.8 and $23.9 \%$ respectively in Shanghai. Community violence was the predominant violence type, affecting $72.8-93.7 \%$ across the sites. More than half of girls (53.7\%) and $45.9 \%$ of boys had at least one adverse mental health outcome. Compared to those that did not report violence, boys exposed to PV had 11.4 higher odds of having a negative perception of health $(95 \% \mathrm{Cl}$ adjusted $\mathrm{OR}=2.45-53.2)$, whilst this figure was 2.58 times in girls $(95 \% \mathrm{Cl}=1.62-4.12)$. Among girls, PV was associated with suicidal thoughts (adjusted $\mathrm{OR}=4.68$; $95 \% \mathrm{Cl}=2.29-9.54)$, posttraumatic stress $(\mathrm{aOR}=4.53 ; 95 \% \mathrm{Cl}=2.44-8.41)$ and depression $(\mathrm{aOR}=2.65 ; 95 \% \mathrm{Cl}=1.25-5$. 63). Among boys, an association was only detected between $\mathrm{PV}$ and depression $(\mathrm{aOR}=1.82 ; 95 \% \mathrm{Cl}=1.00-3.33$ ).

Conclusion: The findings demonstrate that PV is common among both sexes in urban disadvantaged settings across the world, and that it is associated with poor mental health outcomes in girls, and with poor health status in both girls and boys. Clearly, prevention interventions are failing to address violence exposure across multiple contexts, but especially within community settings and in Johannesburg. Interventions are needed to identify adolescents exposed to PV and link them to care, with services targeting a range of mental health conditions among girls and perhaps focusing on depression among boys.
\end{abstract}

Keywords: Polyvictimisation, Mental health, Adolescents, Gender differences, Urban disadvantaged environments

\footnotetext{
* Correspondence: sdelany@wrhi.ac.za

${ }^{1}$ Wits RHI, Faculty of Health Sciences, University of the Witwatersrand,

Johannesburg, South Africa

Full list of author information is available at the end of the article
} 


\section{Background}

Polyvictimisation (PV), or exposure to violence across multiple contexts, causes significant short- and longterm morbidity and even mortality among adolescents [1-3]. Research interest has grown in understanding the impact of multiple exposures to violence across a range of contexts including within the family and community, and from intimate partners and peers $[4,5]$. Globally, rates of exposure to PV in adolescents vary considerably. In high-income countries, for example, estimates of PV range from 10 to $93 \%$ [5-10]. Rates of PV are generally higher in low- and middle-income countries (LMICs) [11], reaching almost 95\% in one community-based sample in Cape Town, South Africa, for example [12].

A constellation of social vulnerabilities means that adolescents living in disadvantaged urban settings are at a particularly high risk for PV [12-15], and there are often very few social and health resources to counter these vulnerabilities $[15,16]$. Victims of PV are at a considerably higher risk for poor mental health, as well as emotional, behavioural and developmental problems compared with those exposed to violence in a single context [17-19]. It has, however, not been clearly established whether the impact of PV on mental health varies by gender in these settings. The burden of $\mathrm{PV}$ and the associated mental health outcomes might be more profound for girls than boys, given high levels of sexual violence against females $[4,20]$. Previously, we showed that adolescent women living in disadvantaged environments experienced a significant burden of gender-based violence and associated health consequences [15]. Equally however, boys may be impacted more by male-on-male physical violence. Studies that have explored differences in prevalence of PV by gender have inconsistent findings [4, 20], suggesting that variations in forms or combinations of violence may create different risk profiles, and that these may be further moderated by age, place of residence and a host of other factors. Multi-site studies, applying standardized survey and analytical methodology, can explore gender differences in detail, and overcome the present problems with generalising findings from studies of single populations. Conclusions that are generalizable are needed to guide broad recommendations for victimisation prevention and care initiatives in disadvantaged urban settings. These, in turn, can then be adapted for implementation in similar settings.

Against this backdrop, we have a unique opportunity to extend the literature by examining gender differences in the association between PV and mental health among adolescents in five economically-distressed urban settings, across three continents. Also, the prevalence of $\mathrm{PV}$, each type of violence and mental health problems in the whole study population are described. This provides useful insights into how adolescents' lived experiences vary in disadvantaged, urban settings across the world.

\section{Methods}

\section{Sample and procedures}

Data were drawn from the cross-sectional Well Being of Adolescents in Vulnerable Environments (WAVE) study, which enrolled adolescents aged 15-19 years living in disadvantaged urban settings in Baltimore (USA), Johannesburg (South Africa), Ibadan (Nigeria), Delhi (India) and Shanghai (China) in 2013. In Shanghai, recruitment was limited to migrant adolescents. Respondent-driven sampling (RDS) was used to recruit approximately 500 participants in each site. All interviews were conducted using computer-assisted self-interview instruments.

Full details of the study methodology [21], measures of violence [15], indicators of mental health and their validity [22], and of the study population [23] are presented in previous publications. All adolescents gave written informed consent/assent. In addition, parents or guardians of adolescents aged 15-17 years gave written informed consent.

\section{Study measures}

The study measures drew on existing validated tools wherever possible. Where such tools could not be located, we developed the tools during a formative study phase that preceded the survey [24]. Polyvictimisation was defined as exposure to two or more types of victimisation in the past 12 months, which consisted of: family violence encompassing the experience or witnessing of at least one act of physical or psychological violence perpetrated by a family member; peer violence including the experience or witnessing of at least one act of physical or psychological violence perpetrated by peers of either sex; community violence, entailing the witnessing of criminal activities, such as drug deals, interpersonal violence with or without a weapon, and arrests, or the experience of having one's house broken into (these measures were drawn from National Survey of American life) $[25,26]$; and intimate partner violence (IPV), which included both physical and sexual violence; and nonpartner sexual violence (NPSV), (i.e., that perpetrated by someone who was not a dating partner or spouse) [15]. IPV, separated into sexual and physical IPV, was assessed only among girls given that experiencing violence from a partner is more common and more severe among women [27]. Given that male IPV was not assessed, boys had a maximum of four types of violence, while girls had a maximum of six. Respondents who had never had an intimate partner were classified as not having experienced IPV.

\section{Mental health outcomes}

Mental health outcomes were identified through selfreported responses to scales assessing symptoms of: posttraumatic stress (Posttraumatic stress disorder 
checklist-civilian version used for diagnosing posttraumatic stress) [28]; depression (the short 10-item version of the Centre for Epidemiological Studies Depression Scale) [29]; and suicidal thoughts in the past 12 months [18]. Ever having planned or attempted suicide was also reported. We also describe the prevalence of: heavy alcohol use (ever having consumed five or more alcohol drinks within $2 \mathrm{~h}$, or being drunk at least once a month [4]); a negative perception of overall health status (responded 'fair' or 'poor' to health status measure); and having sought treatment for mental health problems in the past 12 months.

\section{Data analysis}

Weights were generated via the RDS II estimator [30] to account for inter-cluster correlations. Given differences in the age distribution across centres, a post-stratification age weight was also developed and combined with the RDS weight. Weighted means and percentages were computed for continuous and categorical variables respectively, and compared by gender within each site.

Logistic regression models using weighted data were fitted to evaluate the relationship between PV and perceived health status, posttraumatic stress, depression and suicidal thoughts, separately for males and females. Multivariate logistic regression models were then fitted, again separately by gender, for two-tailed tests of the null hypothesis that odds ratio $(\mathrm{OR})=1$, to evaluate the relationship between between PV and each of the four outcome variables, adjusted for age and city of residence. The reference group in these models for PV was those with no experience of violence and for the city variable, Baltimore. Adjusted ORs (aOR) and their corresponding 95\% confidence intervals were calculated for each study outcome. All analyses used STATA v.12 statistical software.

\section{Results \\ Prevalence of past-year polyvictimisation, by gender within each city}

Past-year PV was extremely common overall, but ranged from a high of $74.5 \%$ of boys and $82.0 \%$ of girls in Johannesburg, to a low of 25.8 and $23.9 \%$ respectively in Shanghai ((Table 1). Though a considerable portion of girls had experienced PV in Delhi (60.5\%) and Ibadan (50.2\%), levels among boys in these settings were even higher (71.0 and $62.5 \%$ respectively). By contrast, levels of PV were notably higher among girls than boys in Baltimore (71.1\% versus $42.5 \%)$, mostly due to high levels of physical IPV experienced by girls (Table 1 ).

When examining the relative frequency of different types of victimisation, the most common was community violence in all cities (range 72.8-93.7\%), except in Shanghai, where peer violence predominated $(61.6 \%$ in boys and $44.3 \%$ in girls; Table 1). Experiences of community violence were higher for adolescent boys than girls in Delhi, Johannesburg and Shanghai. No differences in experience of family violence were observed between adolescent girls and boys in Ibadan, Johannesburg, and Shanghai, while in Delhi and Baltimore, girls reported considerably higher levels of family violence than boys, and as compared with girls elsewhere. A remarkable $40.7 \%$ of girls in Delhi had been physically hurt in their home and $29.6 \%$ had been hurt in a fight with peers (Additional file 1). In Baltimore, girls reported that family violence frequently took the form of being pushed, grabbed, shoved or threatened in their homes, which occurred almost twice as frequently than in boys.

Peer violence was experienced more frequently by adolescent girls in Delhi and Shanghai, compared to boys in those cities (Additional file 2). All forms of sexual IPV were highest among girls in Johannesburg, as was overall occurrence of physical IPV, with almost a third reporting having been slapped and a quarter pushed by an intimate partner. Levels of NPSV were several fold higher in girls than boys in Baltimore, Delhi and Shanghai, while a considerable portion of boys in Johannesburg had experienced this form of violence (34.1\% versus $49.4 \%$ in girls) and it was even more common in boys than girls in Ibadan $(50.0 \%$ versus $26.7 \%$ in girls; Additional file 3).

\section{Prevalence of mental health outcomes, by gender within each city}

Across all sites, posttraumatic stress was considerably more common in adolescent girls than boys, aside for Ibadan where $27.9 \%$ of boys and $22.2 \%$ of girls had posttraumatic stress. Similarly, a higher proportion of adolescent girls than boys reported depressive symptoms in Shanghai (33.5\%), Ibadan (40.2\%) and Johannesburg (46.1\%), which had the highest levels of depression for both sexes. As an exception, boys had higher levels of depression than girls in Baltimore (32.4\% versus 27.3\%). There were also gender differences in suicidal thoughts, plans and attempts across the sites, with a female predominance in Baltimore, Johannesburg and Shanghai. Most especially in Shanghai, the prevalence of suicidal thoughts, plans and attempts was several fold higher among girls than boys. The most consistent differences in mental health outcomes between adolescent boys and girls were observed in Shanghai and Johannesburg, where a higher proportion of girls than boys had each of the mental health outcomes measured.

With regard to co-morbid conditions, heavy alcohol use (assessed among current alcohol users only) was uncommon in Delhi, was considerably more frequent in boys than girls in Ibadan and Johannesburg, and occurred roughly in the same proportion of both sexes 


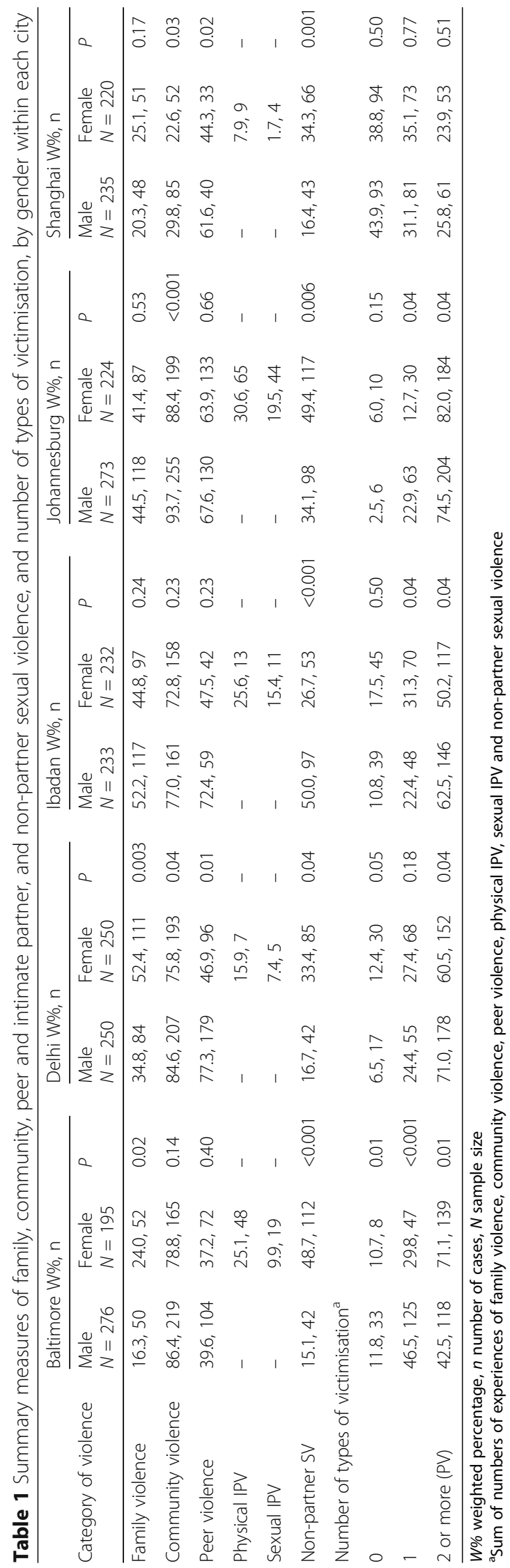


in Baltimore and Shanghai (41 to 48\%). Negative perceptions of overall health status were notably more common in girls than boys in Baltimore (10.1 versus 4.1\%) and Shanghai (16.8\% against 5.2\%). Treatment seeking for mental health concerns was infrequent (around 1\% overall), irrespective of setting (Table 2).

\section{Polyvictimisation and its association with health status and mental health}

The first part of this section presents the findings of the four mental health outcomes as a function of PV, separately for males and females (Tables 3 and 4). The second part sums the associations noted between these outcomes, and age and city (Tables 3 and 4).

For each outcome, the ORs increased for both genders as the number of types of victimisation increased (Tables 3 and 4). There were, however, differences in the magnitude of increase, and statistical significance, according to gender. Moreover, considerable differences were noted between effect sizes in unadjusted and adjusted models.

PV was strongly associated with negative perceptions of overall health for both boys and girls. Specifically, boys that experienced PV had 11.43 higher odds of having a negative perception of health than those who had never experienced violence (95\%CI: 2.45-53.21), whereas these odds were 2.58 in girls with PV (95\%CI: 1.62-4.12; Table 4).

Gender differences were also observed in the association between PV and the mental health outcome variables assessed. Independent of age and city of residence, girls that experienced PV had an increased odds of suicidal thoughts (aOR $=4.68$; 95\%CI: 2.29-9.54), posttraumatic stress $(\mathrm{aOR}=4.53 ; 95 \% \mathrm{CI}: 2.44-8.41)$ and depression $(\mathrm{aOR}=2.65 ; 95 \% \mathrm{CI}: 1.25-5.63)$, while the sizes of point estimates for these three associations were lower among boys. Depression was the only mental health outcome associated with $\mathrm{PV}$ in boys $(\mathrm{aOR}=1.82$; $95 \% \mathrm{CI}=1.00-3.33)$.

Several other findings in the multivariate analysis bear mention. Firstly, associations were detected between having one episode of victimisation and negative perceptions of health for boys ( $\mathrm{aOR}=7.90$; 95\%CI: 1.43-43.56) and suicidal thoughts for girls $(\mathrm{aOR}=2.46$; $95 \% \mathrm{CI}$ : 1.17 5.18) compared to those who had never experienced violence. None of the estimated associations between having one episode of victimisation and the other outcomes were statistically significant, with the point estimates ranging from $0.85-1.41$ ).

Secondly, city of residence was an important determinant of all outcomes assessed, independent of age and of having experienced victimisation. Negative perceptions of health were more common among boys in Johannesburg $(\mathrm{aOR}=3.22$; 95\%CI: 1.63-6.36) and girls in Shanghai $(\mathrm{aOR}=2.45$; 95\%CI: $1.33-4.52)$, than in Baltimore, but less frequent in girls living in Ibadan $(\mathrm{aOR}=0.31$; 95\%CI: 0.13-0.71). Most strikingly, however, being in Johannesburg, as opposed to in Baltimore, was associated with around a two-fold higher odds of all the mental health outcomes, aside from depression in boys. The converse was true of Delhi, where point estimates were the lowest for both genders.

Compared with Baltimore, girls in Ibadan had less posttraumatic stress, but more frequently had depression. Of note, the girls in Shanghai had 2.6 fold higher odds of suicidal thoughts than those in Baltimore (95\%CI: 1.91-3.59), and also had higher odds of posttraumatic stress and depression. In Shanghai, aside from a 1.3 fold higher odds of suicidal thoughts in boys than in Baltimore, no significant differences were detected between boys in these two cities. Finally, age was associated with posttraumatic stress and depression for boys, where ORs of the condition rose with age.

\section{Discussion}

We primarily aimed to explore whether there are gender differences in the association between PV and mental health outcomes among urban disadvantaged adolescents in five cities. In this regard, we demonstrate that whilst past-year PV is remarkably common in adolescents in all five cities, affecting around three quarters of adolescents in four of the five cities, its effects on mental and physical health vary, and differ by gender. PV was strongly associated with negative perceptions of overall health for both boys and girls, but the effect sizes tended to be higher for boys than girls. By contrast, girls that experienced PV had an increased likelihood of suicidal thoughts, posttraumatic stress and depression, while for boys these associations were not significant in adjusted analyses. The point estimates of associations between PV and the mental health outcomes were lower among the boys and not significant aside from depression. We also illustrated clearly that city of residence is an important determinant of all associations studied, even independent of whether boys or girls experienced victimisation.

Most previous studies involved data from one site only and report on single types of victimisation, making it difficult to compare our results to those of others. Where differences are observed between studies, these may also be accounted for by variations in the definition of exposure and outcomes. Nevertheless, our findings are broadly consistent with that of an Italian study among university students, which concluded that PV was associated with a high risk of depression, panic attacks, heavy alcohol use, eating problems, suicidal ideation and attempts, and a negative perception of overall health [4]. Other studies in high-income countries [20] and LMICs [17] have also found that adolescent girls who experienced violence 


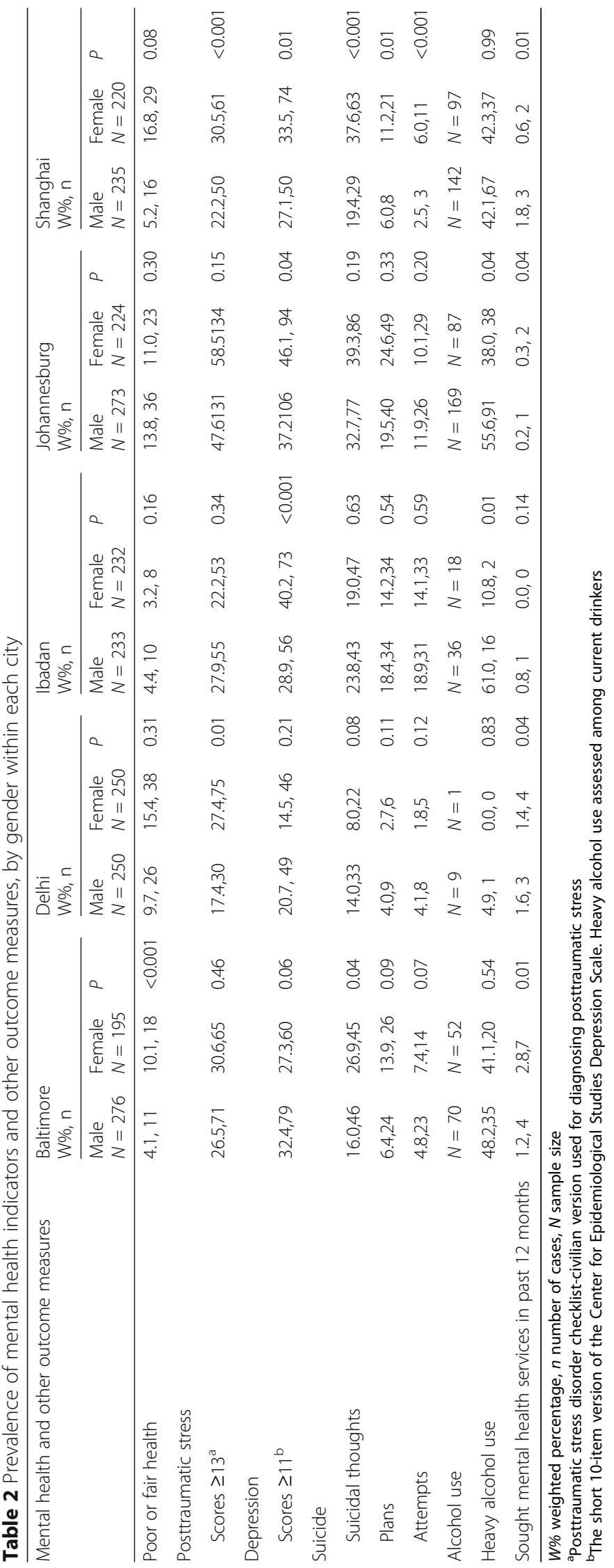


Table 3 Unadjusted odds ratios (with 95\% Cl) for associations between number of types of victimisation and mental health by gender

\begin{tabular}{|c|c|c|c|c|c|c|c|c|}
\hline \multirow{3}{*}{$\begin{array}{l}\text { Independent variables } \\
\text { Number of types of victimisation }\end{array}$} & \multicolumn{4}{|c|}{ Poor or fair health status } & \multicolumn{4}{|c|}{ Posttraumatic stress } \\
\hline & \multicolumn{2}{|l|}{ Male $(n=1208)$} & \multicolumn{2}{|l|}{ Female $(n=1099)$} & \multicolumn{2}{|l|}{ Male $(n=1121)$} & \multicolumn{2}{|l|}{ Female $(n=1069)$} \\
\hline & OR $(95 \% \mathrm{Cl})$ & $P$ & OR $(95 \% \mathrm{Cl})$ & $P$ & OR $(95 \% \mathrm{Cl})$ & $P$ & OR $(95 \% \mathrm{Cl})$ & $P$ \\
\hline 0 (Ref) & 1.00 & & 1.00 & & 1.00 & & 1.00 & \\
\hline 1 & $7.99(2.05,31.25)$ & 0.004 & $1.11(0.46,2.67)$ & 0.811 & $1.63(0.86,3.11)$ & 0.129 & $1.26(0.68,2.34)$ & 0.460 \\
\hline 2 or more (PV) & $12.84(4.21,9.20)$ & $<0.001$ & $1.82(0.80,4.14)$ & 0.149 & $2.29(1.02,5.17)$ & 0.046 & $4.39(2.13,9.04)$ & $<0.001$ \\
\hline \multirow[t]{3}{*}{ R2 value } & 0.02 & & 0.02 & & 0.02 & & 0.06 & \\
\hline & \multicolumn{4}{|l|}{ Depression } & \multicolumn{4}{|l|}{ Suicidal thoughts } \\
\hline & \multicolumn{2}{|l|}{ Male $(n=1151)$} & \multicolumn{2}{|l|}{ Female $(n=1043)$} & \multicolumn{2}{|l|}{ Male $(n=1211)$} & \multicolumn{2}{|l|}{ Female $(n=1102)$} \\
\hline Number of types of victimisation & OR $(95 \% \mathrm{Cl})$ & $P$ & OR $(95 \% \mathrm{Cl})$ & P & OR $(95 \% \mathrm{Cl})$ & P & OR $(95 \% \mathrm{Cl})$ & $P$ \\
\hline 0 (Ref) & 1.00 & & 1.00 & & 1.00 & & 1.00 & \\
\hline 1 & $1.22(0.48,3.08)$ & 0.661 & $0.83(0.58,1.19)$ & 0.304 & $0.83(0.38,1.77)$ & 0.612 & $1.79(0.82,3.92)$ & 0.139 \\
\hline 2 or more (PV) & $1.74(0.94,3.19)$ & 0.074 & $2.12(1.01,4.46)$ & 0.049 & $1.72(0.91,3.25)$ & 0.090 & $2.90(1.06,7.93)$ & 0.039 \\
\hline R2 value & 0.02 & & 0.02 & & 0.02 & & 0.04 & \\
\hline
\end{tabular}

Bold OR values indicate significance at $p<0.05 .95 \% \mathrm{Cl}=95 \%$ confidence interval

were at a higher risk for depression than their male counterparts.

Somewhat unexpectedly, in quite a few instances, boys carried an equal burden of violence and mental illness as girls. This reinforces the importance of overcoming the challenges of reaching men with health services, starting with adolescent boys. Notably, rates of some outcomes among boys escalate rapidly with age.

Addressing the burden of mental illness in adolescents would require site-specific, innovative interventions that are able to take account of the diverse forms of gendered behaviours in different settings. This seems unlikely to occur in the near future, as access to mental health services is dismal for each of the conditions measured and in all sites. With a few exceptions, none of the affected adolescents, whether male or female, are currently receiving treatment. Even if services were available, stigma, especially prevalent among boys, would remain a major obstacle to treatment [31]. Stigma leads to secrecy and avoidance of treatment, and initiatives to counter stigma have met with little success to date [32]. Moreover, as noted in a systematic review, adolescents across the world commonly hold uninformed, negative views of mental health services and professionals, and may believe that accessing such services is a 'sign of weakness' [33]. However, adolescents, especially adolescent boys are proficient in the use of mobile technologies and this has the potential to improve uptake and accessibility of mental health interventions [34].

Regarding city of residence, boys and girls in Johannesburg had higher ORs than Baltimore for almost all indices assessed. In other words, something about living in Johannesburg - other than PV, gender and being disadvantaged - is responsible for the poor mental and physical health in this city. The high levels of PV in
Johannesburg is perhaps not surprising given that South Africa is one of the most violent countries in the world [35]. Living in Delhi, by contrast, appears to be protective against PV and mental illness, though girls in Delhi reported alarming levels of physical violence in the home and from peers. Equally, physical violence from intimate partners affected a quarter to a third of girls in Baltimore, Ibadan and Johannesburg. Variations in violence by gender might partly be explained by differences in gender inequality across sites. Based on the UNDP Gender Inequality Index [36], inequality is highest in Delhi of the cities included in the study, potentially explaining the high levels of family violence among girls in the city. Gender inequalities are also high in Johannesburg, again correlating with high rates of violence among girls and boys in that area. By contrast, in Shanghai, which has the lowest levels of victimisation in the study, inequalities are lowest of the five cities according to the UNDP Index.

Several limitations and strengths of this study should be noted. The cross-sectional design limits the ability to draw causal inferences about the associations detected. We also lack data on the intensity of the individual elements of violence assessed, which implies that severe repeated family violence, for example, would still be counted only as one form of violence. The relatively large samples drawn from five cities across three continents are a strength of the study and increase the validity of the study's assertions. Longitudinal studies could more fully unravel the immediate, underlying and environmental factors that account for PV and its impacts, and identify different patterns of PV and its effects over the life course. Additionally, qualitative analyses could deepen our understanding of how victimisation is experienced by adolescents in different settings [37], and how they develop coping strategies in response. 
Table 4 Odds ratios of associations between polyvictimisation and mental health, by gender, adjusted for age and city of residence

\begin{tabular}{|c|c|c|c|c|c|c|c|c|}
\hline \multirow{3}{*}{$\begin{array}{l}\text { Independent variables } \\
\text { Number of types of victimisation }\end{array}$} & \multicolumn{4}{|c|}{ Poor or fair health status } & \multicolumn{4}{|c|}{ Posttraumatic stress } \\
\hline & \multicolumn{2}{|l|}{ Male $(n=1208)$} & \multicolumn{2}{|l|}{ Female $(n=1099)$} & \multicolumn{2}{|l|}{ Male $(n=1121)$} & \multicolumn{2}{|l|}{ Female $(n=1069)$} \\
\hline & aOR $(95 \% \mathrm{Cl})$ & P & $\mathrm{aOR}(95 \% \mathrm{Cl})$ & P & aOR $(95 \% \mathrm{Cl})$ & $P$ & aOR $(95 \% \mathrm{Cl})$ & $P$ \\
\hline 0 (Ref) & 1.00 & & 1.00 & & 1.00 & & 1.00 & \\
\hline 1 & $7.90(1.43,43.56)$ & 0.019 & $1.35(0.65,2.82)$ & 0.415 & $1.33(0.73,2.42)$ & 0.341 & $1.41(0.78,2.54)$ & 0.248 \\
\hline 2 or more (PV) & $11.43(2.45,53.21)$ & 0.003 & $2.58(1.62,4.12)$ & $<0.001$ & $1.79(0.83,3.88)$ & 0.134 & $4.53(2.44,8.41)$ & $<0.001$ \\
\hline Age (years) & $1.05(0.85,1.30)$ & 0.635 & $0.95(0.85,1.06)$ & 0.325 & $1.20(1.03,1.40)$ & 0.024 & $0.95(0.84,1.09)$ & 0.454 \\
\hline \multicolumn{9}{|l|}{ City of residence } \\
\hline Baltimore (Ref) & 1.00 & & 1.00 & & 1.00 & & 1.00 & \\
\hline Delhi & $2.31(0.77,6.88)$ & 0.128 & $1.61(0.85,3.09)$ & 0.142 & $0.54(0.38,0.78)$ & 0.002 & $0.83(0.60,1.15)$ & 0.256 \\
\hline Ibadan & $0.99(0.48,2.08)$ & 0.988 & $0.31(0.13,0.71)$ & 0.007 & $0.99(0.66,1.51)$ & 0.979 & $0.69(0.48,0.99)$ & 0.046 \\
\hline Johannesburg & $3.22(1.63,6.36)$ & 0.001 & $0.96(0.55,1.68)$ & 0.876 & $2.38(1.50,3.77)$ & 0.001 & $2.66(1.53,4.62)$ & 0.001 \\
\hline Shanghai & $2.04(0.67,6.20)$ & 0.198 & $2.45(1.33,4.52)$ & 0.005 & $0.91(0.64,1.30)$ & 0.596 & $1.68(1.20,2.34)$ & 0.003 \\
\hline \multirow[t]{3}{*}{ R2 value } & 0.05 & & 0.06 & & 0.07 & & 0.09 & \\
\hline & \multicolumn{4}{|l|}{ Depression } & \multicolumn{4}{|l|}{ Suicidal thoughts } \\
\hline & \multicolumn{2}{|l|}{ Male $(n=1151)$} & \multicolumn{2}{|l|}{ Female $(n=1043)$} & \multicolumn{2}{|l|}{ Male $(n=1211)$} & \multicolumn{2}{|l|}{ Female $(n=1102)$} \\
\hline Number of types of victimisation & $\mathrm{aOR}(95 \% \mathrm{Cl})$ & P & $\mathrm{aOR}(95 \% \mathrm{Cl})$ & $P$ & $\mathrm{aOR}(95 \% \mathrm{Cl})$ & $P$ & $\mathrm{aOR}(95 \% \mathrm{Cl})$ & $P$ \\
\hline 0 (Ref) & 1.00 & & 1.00 & & 1.00 & & 1.00 & \\
\hline 1 & $1.15(0.48,2.77)$ & 0.747 & $0.96(0.65,1.40)$ & 0.819 & $0.85(0.41,1.74)$ & 0.640 & $2.46(1.17,5.18)$ & 0.019 \\
\hline 2 or more (PV) & $1.82(1.00,3.33)$ & 0.050 & $2.65(1.25,5.63)$ & 0.013 & $1.65(0.88,3.11)$ & 0.116 & $4.68(2.29,9.54)$ & $<0.001$ \\
\hline Age (years) & $1.19(1.01,1.39)$ & 0.034 & $1.05(0.85,1.29)$ & 0.641 & $0.93(0.82,1.05)$ & 0.220 & $0.98(0.84,1.15)$ & 0.831 \\
\hline \multicolumn{9}{|l|}{ City of residence } \\
\hline Baltimore (Ref) & 1.00 & & 1.00 & & 1.00 & & 1.00 & \\
\hline Delhi & $0.49(0.31,0.76)$ & 0.002 & $0.44(0.34,0.55)$ & $<0.001$ & $0.68(0.40,1.16)$ & 0.151 & $0.22(0.19,0.27)$ & $<0.001$ \\
\hline Ibadan & $0.73(0.57,0.95)$ & 0.019 & $1.97(1.49,2.61)$ & $<0.001$ & $1.37(0.84,2.23)$ & 0.193 & $0.68(0.40,1.15)$ & 0.142 \\
\hline Johannesburg & $1.09(0.80,1.50)$ & 0.571 & $1.94(1.50,2.52)$ & $<0.001$ & $2.00(1.34,2.99)$ & 0.001 & $1.49(1.04,2.14)$ & 0.030 \\
\hline Shanghai & $0.88(0.69,1.12)$ & 0.276 & $1.94(1.39,2.70)$ & $<0.001$ & $1.30(1.02,1.66)$ & 0.033 & $2.62(1.91,3.59)$ & $<0.001$ \\
\hline R2 value & 0.04 & & 0.05 & & 0.04 & & 0.10 & \\
\hline
\end{tabular}

Bold OR values indicate significance at $p<0.05$

$a O R$ adjusted odds ratios, $95 \% \mathrm{Cl} 95 \%$ confidence interval

\section{Conclusions}

In conclusion, this study sheds some light on the role of gender in the intersections between PV and mental health among adolescents in five urban disadvantaged settings. The results show that PV is associated with the mental health outcomes for girls, and poor selfrated health and depression for the boys. Girls who have experienced PV require specific targeting with services that address posttraumatic stress and depression. Overall, the findings highlight the dire need for interventions that reduce violence exposure and improve safety in these settings. For instance, there is evidence from Uganda, which shows the potential to reduce violence by focussing on social mobilisation [38]. It is also possible to modify violence outcomes within programmatic time frames when interventions are delivered at a structural level [39]. mHealth interventions for adolescents show much promise, such as the rapidly expanding Circle of Six smartphone application which addresses gender-based violence [27]. Historically, intervention systems address specific forms of violence in general - for example, IPV support, family violence support etc., but rarely consider the overlap between these [16].

This study highlights the need to shift this approach to one which understands and addresses the interacting compounding impacts of violence exposure across multiple contexts. Moreover, high levels of PV indicate that current preventive efforts are poorly, if at all, effective, and the effects of this are magnified by the absence of treatment for the mental health consequences thereof. This highlights the critical importance of identifying new funding for interventions to prevent victimisation across multiple contexts and to mitigate its short- and long-term consequences among adolescents of both sexes in these settings. 


\section{Additional files}

Additional file 1: Table S1. Prevalence of family and community violence, by gender within each city. (DOCX 36 kb)

Additional file 2: Table S2. Prevalence of peer violence, by gender within each city. (DOCX $32 \mathrm{~kb}$ )

Additional file 3: Table S3. Prevalence of intimate partner and nonpartner sexual violence, by gender within each city. (DOCX 35 kb)

\section{Abbreviations}

IPV: Intimate partner violence; LMIC: Low- and Middle-Income Countries; NPSV: Non-partner sexual violence; PV: Polyvictimisation; RDS: Respondentdriven Sampling; UNDP: United Nations Development Programme; WAVE: Wellbeing of Adolescents in Vulnerable Environments Study

\section{Acknowledgments}

The authors would like to acknowledge the contribution of participants and the WAVE study teams in each of the five cities. This research was supported by Young Health Programme, a partnership between AstraZeneca, Johns Hopkins Bloomberg School of Public Health, and Plan International, a leading global children's charity.

\section{Funding}

This research was supported by Young Health Programme, a partnership between AstraZeneca, Johns Hopkins Bloomberg School of Public Health and Plan International, a leading global children's charity. In Ibadan, the study was funded by The Bill and Melinda Gates Institute at Johns Hopkins Bloomberg School of Public Health through its funding to The Centre for Population and Reproductive Health, University of Ibadan. MK and SDM were supported in part by UKaid from the Department for International Development through the STRIVE Research Programme Consortium (Ref: Po 5244). However, the views expressed do not necessarily reflect the department's official policies. WRHI Research Capacity Building funds were used to fund the publications charges for the supplement.

\section{Availability of data and materials}

The datasets during and/or analysed during the current study are available from the corresponding author on reasonable request.

\section{Authors' contributions}

MRD, AO, RA, YC, HB and SDM planned the study, developed the study methods and interview guides, and led implementation of the study. MK PTP, MFC and SDM developed the analysis plan, analysed the data, and drafted the manuscript. All authors critically revised the draft of the manuscript and gave approval of the final manuscript.

\section{Competing interests}

The authors declare that they have no competing interests.

\section{Consent for publication}

Not applicable.

\section{Ethics approval and consent to participate}

The study received approval from the Committee on Human Research at the Johns Hopkins University and ethics review committees in all collaborating sites.

\section{About this supplement}

This article has been published as part of BMC Public Health Volume 17 Supplement 3, 2017: Urban Health at the Edge: A Series on Reproductive Health and HIV in inner-city Johannesburg. The full contents of the supplement are available online at https://bmcpublichealth.biomedcentral.com/articles/supplements/volume-17-supplement-3.

\section{Publisher's Note}

Springer Nature remains neutral with regard to jurisdictional claims in published maps and institutional affiliations.

\section{Author details}

${ }^{1}$ Wits RHI, Faculty of Health Sciences, University of the Witwatersrand, Johannesburg, South Africa. ${ }^{2}$ Africa Centre for Migration and Society, University of the Witwatersrand, Johannesburg, South Africa. ${ }^{3}$ Department of Population, Family and Reproductive Health, Johns Hopkins Bloomberg School of Public Health, Baltimore, MD, USA. Institute of Child Health, College of Medicine, University of Ibadan/University College Hospital Ibadan, Ibadan, Nigeria. ${ }^{5}$ Population Council, New Delhi, India. ${ }^{6}$ Shanghai Institute of Planned Parenthood Research, Shanghai, China. ${ }^{7}$ Family Planning NSW, Sydney, Australia.

Published: 4 July 2017

\section{References}

1. Finkelhor D, Ormrod RK, Turner HA. Poly-victimization: a neglected component in child victimization. Child Abuse Negl. 2007;31(1):7-26.

2. Finkelhor D, Ormrod RK, Turner HA. Polyvictimization and trauma in a national longitudinal cohort. Dev Psychopathol. 2007;19(1):149-66.

3. Finkelhor D, Turner HA, Shattuck A, Hamby SL. Violence, crime, and abuse exposure in a national sample of children and youth: an update. JAMA Pediatr. 2013;167(7):614-21.

4. Romito P, Grassi M. Does violence affect one gender more than the other? The mental health impact of violence among male and female university students. Soc Sci Med. 2007:65(6):1222-34.

5. Turner HA, Finkelhor D, Ormrod R. Poly-victimization in a national sample of children and youth. Am J Prev Med. 2010;38(3):323-30.

6. Margolin G, Vickerman KA, Oliver PH, Gordis EB. Violence exposure in multiple interpersonal domains: cumulative and differential effects. J Adolesc Health. 2010;47(2):198-205.

7. Mrug S, Loosier PS, Windle M. Violence exposure across multiple contexts: individual and joint effects on adjustment. Am J Orthop. 2008;78(1):70-84.

8. Ellonen N, Salmi V. Poly-victimization as a life condition: correlates of polyvictimization among Finnish children. J Scand Stud Criminology Crime Prev. 2011;12(01):20-44

9. Pereda N, Guilera G, Abad J. Victimization and polyvictimization of Spanish children and youth: results from a community sample. Child Abuse Negl. 2014;38(4):640-9.

10. Kennedy AC. An ecological approach to examining cumulative violence exposure among urban, African American adolescents. Child Adolesc Soc Work J. 2008;25(1):25-41.

11. Le MT, Holton S, Romero L, Fisher J. Polyvictimization among children and adolescents in low- and lower-middle-income countries: a systematic review and meta-analysis. Trauma Violence Abuse. 2016.

12. Kaminer D, du Plessis B, Hardy A, Benjamin A. Exposure to violence across multiple sites among young South African adolescents. Peace Conflict. 2013, 19(2):112.

13. Turner HA, Shattuck A, Finkelhor D, Hamby S. Polyvictimization and youth violence exposure across contexts. J Adolesc Health. 2016:58(2):208-14.

14. Kaminer D, Hardy A, Heath K, Mosdell J, Bawa U. Gender patterns in the contribution of different types of violence to posttraumatic stress symptoms among South African urban youth. Child Abuse Negl. 2013:37(5):320-30

15. Decker MR, Peitzmeier S, Olumide A, Acharya R, Ojengbede O, Covarrubias L, Gao E, Cheng Y, Delany-Moretlwe S, Brahmbhatt H. Prevalence and Health Impact of Intimate Partner Violence and Non-partner Sexual Violence Among Female Adolescents Aged 15-19 Years in Vulnerable Urban Environments: A Multi-Country Study. J Adolesc Health. 2014;55(6):S58-S67.

16. Decker MR, Benning L, Weber KM, Sherman SG, Adedimeji A, Wilson TE, Cohen J, Plankey MW, Cohen MH, Golub ET. Physical and sexual violence predictors: 20 years of the women's interagency HIV study cohort. Am J Prev Med. 2016:51(5):731-42.

17. du Plessis B, Kaminer D, Hardy A, Benjamin A. The contribution of different forms of violence exposure to internalizing and externalizing symptoms among young South African adolescents. Child Abuse Negl. 2015;45:80-9.

18. Collings SJ, Penning SL, Valjee SR. Lifetime Poly-Victimization and Posttraumatic Stress Disorder among School-Going Adolescents in Durban, South Africa. J Psychiatry. 2014;17:133. doi:10.4172/Psychiatry.1000133.

19. Cyr K, Clement ME, Chamberland C. Lifetime prevalence of multiple victimizations and its impact on children's mental health. J Interpers Violence. 2014;29(4):616-34. 
20. Romito $P$, Beltramini L, Escribà-Agüir V. Intimate partner violence and mental health among Italian adolescents: gender similarities and differences. Violence Against Women. 2013;19(1):89-106.

21. Decker MR, Marshall BD, Emerson M, Kalamar A, Covarrubias L, Astone N, Wang Z, Gao E, Mashimbye L, Delany-Moretlwe S, et al. Respondent-driven sampling for an adolescent health study in vulnerable urban settings: a multi-country study. J Adolesc Health. 2014;55(6 Supplement):S6-S12.

22. Cheng Y, Li X, Lou C, Sonenstein FL, Kalamar A, Jejeebhoy S, DelanyMoretlwe S, Brahmbhatt $\mathrm{H}$, Olumide AO, Ojengbede O. The association between social support and mental health among vulnerable adolescents in five cities: findings from the study of the well-being of adolescents in vulnerable environments. J Adolesc Health. 2014;55(6 Supplement):S31-8.

23. Olumide $A O$, Robinson $A C$, Levy PA, Mashimbye L, Brahmbhatt $H$, Lian Q, Ojengbede O, Sonenstein FL, Blum RW. Predictors of substance use among vulnerable adolescents in five cities: findings from the wellbeing of adolescents in vulnerable environments study. J Adolesc Health. 2014;55(6):S39-47.

24. Mmari K, Blum R, Sonenstein F, Marshall B, Brahmbhatt H, Venables E, Delany-Moretlwe S, Lou C, Gao E, Acharya R, et al. Adolescents' perceptions of health from disadvantaged urban communities: findings from the WAVE study. Soc Sci Med. 2014;104:124-32.

25. Mmari K, Lantos H, Blum RW, Brahmbhatt H, Sangowawa A, Yu C, DelanyMoretlwe S. A global study on the influence of neighborhood contextual factors on adolescent health. J Adolesc Health. 2014;55(6 Suppl):S13-20.

26. Jackson JS, Torres M, Caldwell CH, Neighbors HW, Nesse RM, Taylor RJ, Trierweiler SJ, Williams DR. The National Survey of American life: a study of racial, ethnic and cultural influences on mental disorders and mental health. Int J Methods Psychiatr Res. 2004;13(4):196-207.

27. Stockl H, Devries K, Rotstein A, Abrahams N, Campbell J, Watts C, Moreno CG. The global prevalence of intimate partner homicide: a systematic review. Lancet. 2013;382(9895):859-65.

28. Blanchard EB, Jones-Alexander J, Buckley TC, Forneris CA. Psychometric properties of the PTSD checklist (PCL). Behav Res Ther. 1996;34(8):669-73.

29. Radloff LS. The use of the Center for Epidemiologic Studies Depression Scale in adolescents and young adults. J Youth Adolesc. 1991;20(2):149-66.

30. Spiller M, Cameron C, Heckathorn DD. Respondent-driven sampling analysis tool User's guide - version 7.1. Ithaca: Cornell University; 2012.

31. Kaushik A, Kostaki E, Kyriakopoulos M. The stigma of mental illness in children and adolescents: a systematic review. Psychiatry Res. 2016;243:469-94.

32. Rickwood D, Cavanagh S, Curtis L, Sakrouge R, Roberts MC, Beidleman WB, Wurtele SK. Educating young people about mental health and mentalillness: evaluating a school-based programme. Int J Ment Health Promot. 2004:6:23-32.

33. Goodwin J, Savage E, Horgan A. Adolescents' and young Adults' beliefs about mental health services and care: a systematic review. Arch Psychiatr Nurs. 2016;30(5):636-44.

34. Hollis C, Falconer CJ, Martin JL, Whittington C, Stockton S, Glazebrook C, Davies EB. Annual research review: Digital health interventions for children and young people with mental health problems: a systematic and metareview. J Child Psychol Psychiatry. 2017;58(4):474-503.

35. Wikipedia: List of countries by intentional homicide rate. 2016.

36. UNDP. Gender inequality Index. Geneva: United Nations Development Program; 2012.

37. White JW. A gendered approach to adolescent dating violence: conceptual and methodological issues. Pyschology of Women Quarterly. 2009;33(1):1-15.

38. Abramsky T, Devries KM, Michau L, Nakuti J, Musuya T, Kiss L, Kyegombe N, Watts C. Ecological pathways to prevention: how does the SASA! Community mobilisation model work to prevent physical intimate partner violence against women? BMC Public Health. 2016;16:339.

39. Ellsberg M, Arango DJ, Morton M, Gennari F, Kiplesund S, Contreras M, Watts C. Prevention of violence against women and girls: what does the evidence say? Lancet. 385(9977):1555-66.

\section{Submit your next manuscript to BioMed Central and we will help you at every step:}

- We accept pre-submission inquiries

- Our selector tool helps you to find the most relevant journal

- We provide round the clock customer support

- Convenient online submission

- Thorough peer review

- Inclusion in PubMed and all major indexing services

- Maximum visibility for your research

Submit your manuscript at www.biomedcentral.com/submit
Biomed Central 